Alma Mater Studiorum - Università di Bologna DEPARTMENT OF ECONOMICS

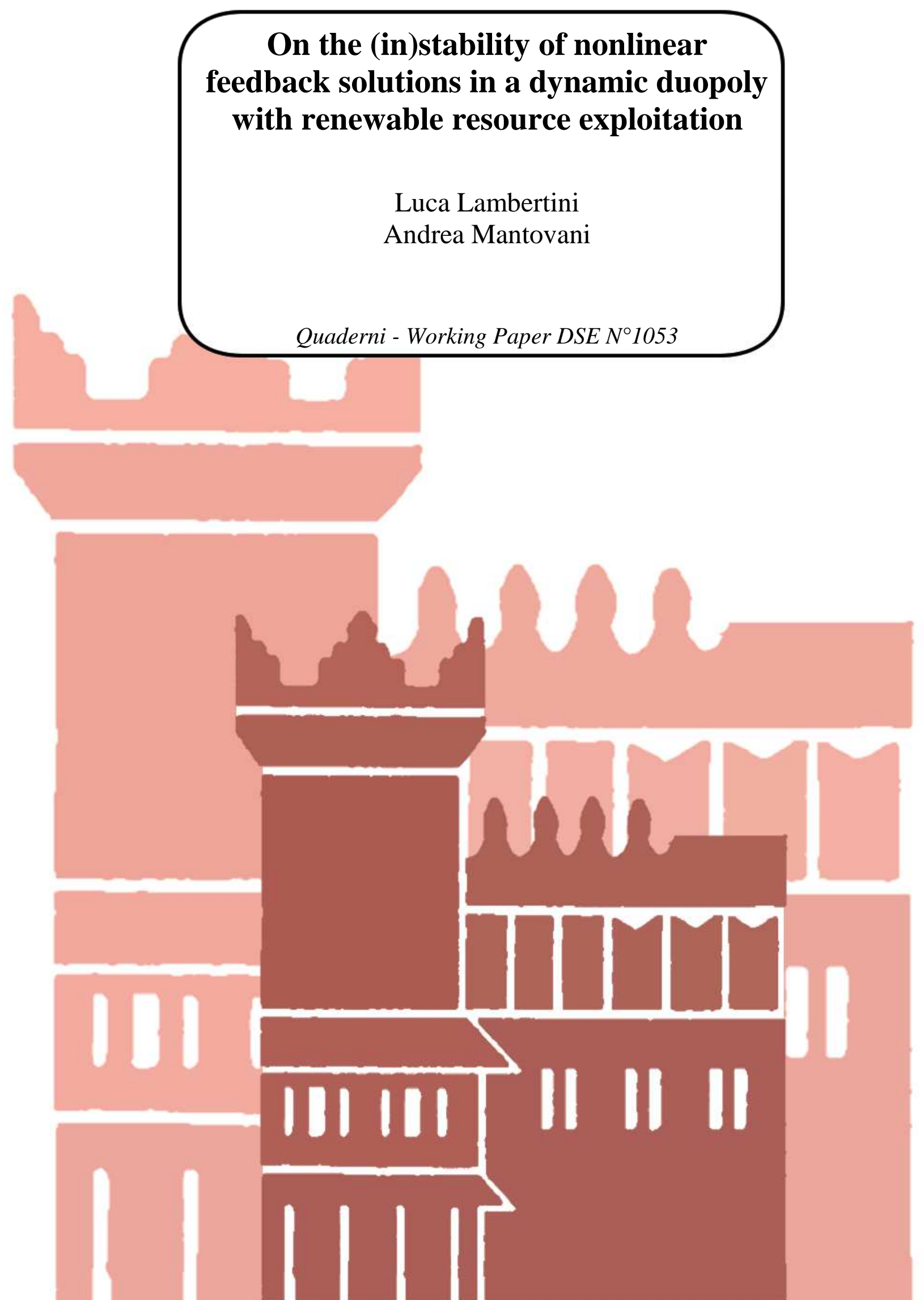




\title{
On the (in)stability of nonlinear feedback solutions in a dynamic duopoly with renewable resource exploitation
}

\author{
Luca Lambertini and Andrea Mantovani \\ Department of Economics, University of Bologna \\ Strada Maggiore 45, 40125 Bologna, Italy \\ luca.lambertini@unibo.it; a.mantovani@unibo.it
}

February 10, 2016

\begin{abstract}
We revisit Fujiwara's (2008) differential duopoly game to show that the degenerate nonlinear feedback identified by the tangency point with the stationary state line is indeed unstable, given the dynamics of the natural resource exploited by firms. To do so, we fully characterise the continuum of nonlinear feedback solution via Rowat's (2007) method, characterising the infinitely many stable nonlinear feedback equilibria.
\end{abstract}

JEL codes: C73, L13, Q2

Keywords: differential games; renewable resources; feedback strategies 


\section{Introduction}

We revisit Fujiwara's (2008) model of dynamic duopolistic exploitation of a renewable resource. In his note, Fujiwara characterises, amongst other features, the nonlinear feedback solution at the tangency point between the steady state locus of the natural resource and the map of firms' isoclines. In doing so, Fujiwara (2008, p. 219) states the following: "Rowat (2007, pp. 3193-3194) gives useful conditions for [the nonlinear feedback control] to be an equilibrium strategy. At first sight, they are violated in the present model but such a guess is incorrect since Rowat (2007) assumes that player i's payoff does not depend on its rival's strategy".

The purpose of our rejoinder is (i) to show that the tangency solution is indeed unstable due to the dynamic properties of the model, and (ii) to characterise the continuum of stable nonlinear feedback equilibria identified by the appropriate intersections between isoclines and the steady state locus. Each of the equilibria belonging to this set can be reached provided that the initial stock of the resource is low enough. To this aim, we strictly follow Rowat's (2007) procedure. ${ }^{1}$

The remainder of the paper is organised as follows. Section 2 illustrates the setup. The linear feedback solution is illustrated in section 3. Nonlinear feedback strategies are dealt with in section 4 . Concluding remarks are in section 5 .

\footnotetext{
${ }^{1}$ Rowat (2007) uses a model where players have quadratic loss functions which are additively separable w.r.t. players' controls. This has the consequence that instantaneous best replies in the control space are orthogonal to each other, which is the source of Fujiwara's observation. However, this feature is altogether unrelated to the stability issue, as the ensuing analysis is about to illustrate.
} 


\section{The model}

The setup is the same as in Benchekroun (2003), Fujiwara (2008) and Colombo and Labrecciosa (2015). The model illustrates a differential oligopoly game of resource extraction unravelling over continuous time $t \in[0, \infty)$. The market is supplied by two fully symmetric firms ${ }^{2}$ producing a homogeneous good, whose inverse demand function is $p=a-X$ at any time $t$, with $X=\sum_{i=1}^{2} x_{i}$. Firms share the same technology, characterised by marginal cost $c \in(0, a)$, constant over time. The individual instantaneous profit function is $\pi_{i}=(p-c) x_{i}$. Firms exploit a common pool renewable resource, whose evolution over time is described by the following dynamics:

$$
\dot{S}=F(S)-X
$$

with

$$
F(S)=\left\{\begin{array}{c}
k S \forall S \in\left(0, S_{y}\right] \\
k S_{y}\left(\frac{S_{\max }-S}{S_{\max }-S_{y}}\right) \forall S \in\left(S_{y}, S_{\max }\right]
\end{array}\right.
$$

where $S$ is the resource stock, $k>0$ is its implicit growth rate when the stock is at most equal to $S_{y}$ and $k S_{y}$ is the maximum sustainable yield. Taken together, (1-2) imply that (i) if the resource stock is sufficiently small the population grows at an exponential rate; and (ii) beyond $S_{y}$, the asset grows at a decreasing rate. Moreover, $S_{\max }$ is the carrying capacity of the habitat, beyond which the growth rate of the resource is negative, being limited by available amounts of food and space. In the remainder, we will confine our attention to the case in which $F(S)=k S$, as in Fujiwara (2008).

Firms play noncooperatively and choose their respective outputs simultaneously at every instant. In the remainder, in order to save upon notation,

\footnotetext{
${ }^{2}$ The analysis of oligopolistic interaction in the same setup is in Benchekroun (2008) and Lambertini and Mantovani (2014).
} 
we will pose $\sigma \equiv a-c>0$. The $i$-th firm maximises the discounted profit flow

$$
\Pi_{i}=\int_{0}^{\infty} \pi_{i} e^{-r t} d t=\int_{0}^{\infty}\left(\sigma-x_{i}-x_{j}\right) x_{j} e^{-r t} d t,
$$

under the constraint posed by the state equation

$$
\dot{S}=k S-X
$$

The initial condition is $S(0)=S_{0}>0$. Parameter $r>0$ is the discount rate, common to all managers and constant over time. To guarantee the positivity of the residual resource stock at the steady state under linear feedback strategies, the ensuing analysis will be carried out under the following assumption (cf. Fujiwara, 2008, p. 218):

\section{Assumption $1 k>5 r / 2$.}

If firms don't internalise the consequences of their behaviour at any time and play the individual (static) Cournot-Nash output $x^{C N}=\sigma / 3$ at all times, then the residual amount of the natural resource in steady state is $S^{C N}=2 \sigma /(3 k)=X^{C N} / k$. As the ensuing analysis illustrates, the static solution indeed corresponds to the open-loop one, which is unstable.

\section{The linear feedback solution}

The Hamilton-Jacobi-Bellman (HJB) of firm $i$ is

$$
r V_{i}(S)=\max _{x_{i}}\left[(\sigma-X) x_{i}+V_{i}^{\prime}(S)(k S-X)\right]
$$

where $V_{i}(S)$ is firm $i$ 's value function, and $V_{i}^{\prime}(S)=\partial V_{i}(S) / \partial S$. The first order condition (FOC) on $x_{i}$ is

$$
\sigma-2 x_{i}-x_{j}-V_{i}^{\prime}(S)=0
$$


In view of the ex ante symmetry across firms, we impose the symmetry conditions $x_{i}=x(S)$ and $V_{i}(S)=V(S)$ for all $i$ and solve FOC (6) to obtain

$$
x^{F}(S)=\max \left\{0, \frac{\sigma-V^{\prime}(S)}{3}\right\}
$$

where superscript $F$ stands for feedback. Consider the case where $\sigma-$ $V^{\prime}(S)>0$. Substituting $x^{F}(S)=\left(\sigma-V^{\prime}(S)\right) / 3$ into (5), the latter can be rewritten as follows:

$$
\frac{\sigma\left[5 V^{\prime}(S)-\sigma\right]+9\left[r V(S)-k S V^{\prime}(S)\right]-4 V^{\prime}(S)^{2}}{9}=0
$$

Then, we may pose $V(S)=\epsilon_{1} S^{2}+\epsilon_{2} S+\epsilon_{3}$, so that $V^{\prime}(S)=2 \epsilon_{1} S+\epsilon_{2}$. From (8) we obtain the following system of Riccati equations:

$$
\begin{gathered}
\epsilon_{1}\left[9(r-2 k)-16 \epsilon_{1}\right]=0 \\
10 \epsilon_{1} \sigma+\epsilon_{2}\left[9(r-k)-16 \epsilon_{1}\right]=0 \\
9 \epsilon_{3} r+\epsilon_{2}\left[5 \sigma-4 \epsilon_{2}\right]-\sigma^{2}=0
\end{gathered}
$$

Equations (10-11) are solved by

$$
\begin{aligned}
\epsilon_{3} & =\frac{\sigma^{2}-\epsilon_{2}\left[5 \sigma-4 \epsilon_{2}\right]}{9 r} \\
\epsilon_{2} & =\frac{9 \epsilon_{3} r}{9(k-r)+16 \epsilon_{1}}
\end{aligned}
$$

while the roots of (9) are

$$
\epsilon_{11}=0 ; \epsilon_{12}=\frac{9(r-2 k)}{16}
$$

whereby, if $\epsilon_{1}=\epsilon_{11}$, the individual equilibrium output is $x^{F 1}(S)=x^{C N}=$ $x^{O L}$, i.e., the open-loop production level, ${ }^{3}$ while if $\epsilon_{1}=\epsilon_{12}$, the individual equilibrium output is

$$
x^{F 2}(S)=\frac{\sigma(5 r-2 k)+9(2 k-r) k S}{24 k}
$$

\footnotetext{
${ }^{3}$ That is, here the open-loop solution is a degenerate feedback one. For more on games with this feature, see Fershtman (1987), Mehlmann (1988, ch. 4), Dockner et al. (2000, ch. 7) and Cellini et al. (2005), inter alia.
} 
If $x=x^{F 2}(S)$, the steady state level of the natural resource stock is

$$
S^{F 2}=\frac{\sigma(2 k-5 r)}{3 k(2 k-3 r)}>0
$$

for all values of $k$ satisfying Assumption 1. In the remainder, we define $S^{F 2} \equiv S^{L F}$ and $x^{F 2}\left(S^{L F}\right)=x^{L F}$.

\section{Nonlinear feedback equilibria}

To characterise the continuum of nonlinear feedback solutions, we adopt the same procedure as in Rowat (2007), ${ }^{4}$ again confining to symmetric equilibria. Imposing the symmetry condition $x_{i}=x(S)$ for all $i$ and solving (6), we obtain $V^{\prime}(S)=\sigma-3 x(S)$. Substituting this into (5), differentiating both sides with respect to $S$ and rearranging, any feedback strategy is implicitly identified by

$$
x^{\prime}(S)=\frac{(k-r)[\sigma-3 x(S)]}{\sigma+3 k S-8 x(S)},
$$

which must hold together with the terminal condition $\lim _{t \rightarrow \infty} e^{-r t} V(s)=0$. From (16) we see that

$$
\begin{gathered}
x^{\prime}(S)=0 \Leftrightarrow x_{0}(S)=\frac{\sigma}{3}=x^{F 1}(S)=x^{O L} \\
x^{\prime}(S) \rightarrow \pm \infty \Leftrightarrow x_{\infty}(S)=\frac{\sigma+3 k S}{8}
\end{gathered}
$$

Since the feedback control $x^{F}(S)$ is (7), the HJB equation is $r V(S)-$ $k S V^{\prime}(S)=0$ for all $\sigma \in\left(0, V^{\prime}(S)\right]$. In such a case, $V(S)=S^{r / k} \boldsymbol{C}$, provided $\boldsymbol{C}>k \sigma S^{\frac{k-r}{k}} / r$. Otherwise, the HJB equation is (8), whereby

$$
r V(S)=\frac{4 V^{\prime}(S)^{2}+\sigma^{2}+V^{\prime}(S)(9 k S-5 \sigma)}{9}
$$

\footnotetext{
${ }^{4}$ Nonlinear feedback solutions have been investigated in oligopoly theory, environmental and resource economics and other fields. See Tsutsui and Mino (1990), Shimomura (1991), Dockner and Sorger (1996), Itaya and Shimomura (2001), Rubio and Casino (2002) and Colombo and Labrecciosa (2015), inter alia.
} 
(19) can be differentiated w.r.t. $S$ on both sides, to obtain

$$
w^{\prime}(S)=\frac{9(k-r) w(S)}{5(\sigma-k S)-4 w(S)}
$$

where $w(S) \equiv V^{\prime}(S)$ and $\sigma \neq[5 k S+4 w(S)] / 5$. Equation (20) can be usefully rewritten through a transformation of variables using $w=\varkappa+A$ and $S=\psi+B$, with $A=0$ and $B=5 \sigma /(9 k)$, into

$$
\frac{d \varkappa}{d \psi}=\frac{5(r-k) \varkappa}{9 k \psi+8 \varkappa}
$$

which, defining $z \equiv \varkappa / \psi$, whereby $\varkappa=\psi z$ and $\partial \varkappa / \partial \psi=z+\psi \cdot \partial z / \partial \psi$, rewrites as

$$
z+\psi \frac{\partial z}{\partial \psi}=\frac{5(r-k) z}{9 k+8 z}
$$

This has two constant solutions, $z_{\mathbf{a}}=0$ and $z_{\mathbf{b}}=9(r-2 k) / 8$. Going back to the original control variables, we have

$$
x_{\mathbf{a}}=\frac{\sigma-A-z_{\mathbf{a}}(S-B)}{3} ; x_{\mathbf{b}}=\frac{\sigma-A-z_{\mathbf{b}}(S-B)}{3}
$$

where $A$ and $B$ are known. The levels of $S$ solving $\dot{S}=0$ at $x=x_{\mathbf{a}}$ and $x=x_{\mathbf{b}}$ are, respectively,

$$
S_{\mathbf{a}}=\frac{2 \sigma}{3 k} ; S_{\mathbf{b}}=\frac{5 \sigma}{3 k}
$$

with $S_{\mathbf{b}}<B$ since $k>5 r / 2>3 r / 2$. Then, for $z \neq\left\{z_{\mathbf{a}}, z_{\mathbf{b}}\right\}$, one can solve

$$
\frac{d \psi}{\psi}=\frac{[9 k / 8+z] d z}{\left(z-z_{\mathbf{a}}\right)\left(z-z_{\mathbf{b}}\right)}=\frac{\gamma_{\mathbf{a}} d z}{\left(z-z_{\mathbf{a}}\right)}+\frac{\gamma_{\mathbf{b}} d z}{\left(z-z_{\mathbf{b}}\right)}
$$

where coefficients $\gamma_{\mathbf{a}}=k /(2 k-r)$ and $\gamma_{\mathbf{b}}=(k-r) /(2 k-r)$ are univocally determined through the method of partial fractions. Integrating (25), we get $\ln |\psi|=\widetilde{\boldsymbol{M}}+\gamma_{\mathbf{a}} \ln \left|z-z_{\mathbf{a}}\right|+\gamma_{\mathbf{b}} \ln \left|z-z_{\mathbf{b}}\right|$, where $\widetilde{\boldsymbol{M}}$ is an integration constant. Exponentiation yields $|\psi|=\left(\left|z-z_{\mathbf{a}}\right|^{\gamma_{\mathbf{a}}}\left|z-z_{\mathbf{b}}\right|^{\gamma_{\mathbf{b}}}\right) / \boldsymbol{M}$, with $\boldsymbol{M}=$ $e^{-\widetilde{M}}$ and $\boldsymbol{M}$ can be rewritten in terms of $w(S)$ and $S$ as follows:

$$
\boldsymbol{M}=\left[w(S)-A-S_{\mathbf{a}}(S-B)\right]^{\gamma_{\mathbf{a}}} \cdot\left[w(S)-A-S_{\mathbf{b}}(S-B)\right]^{\gamma_{\mathbf{b}}} .
$$


Figure 1 Linear and nonlinear feedback solutions in the state-control space

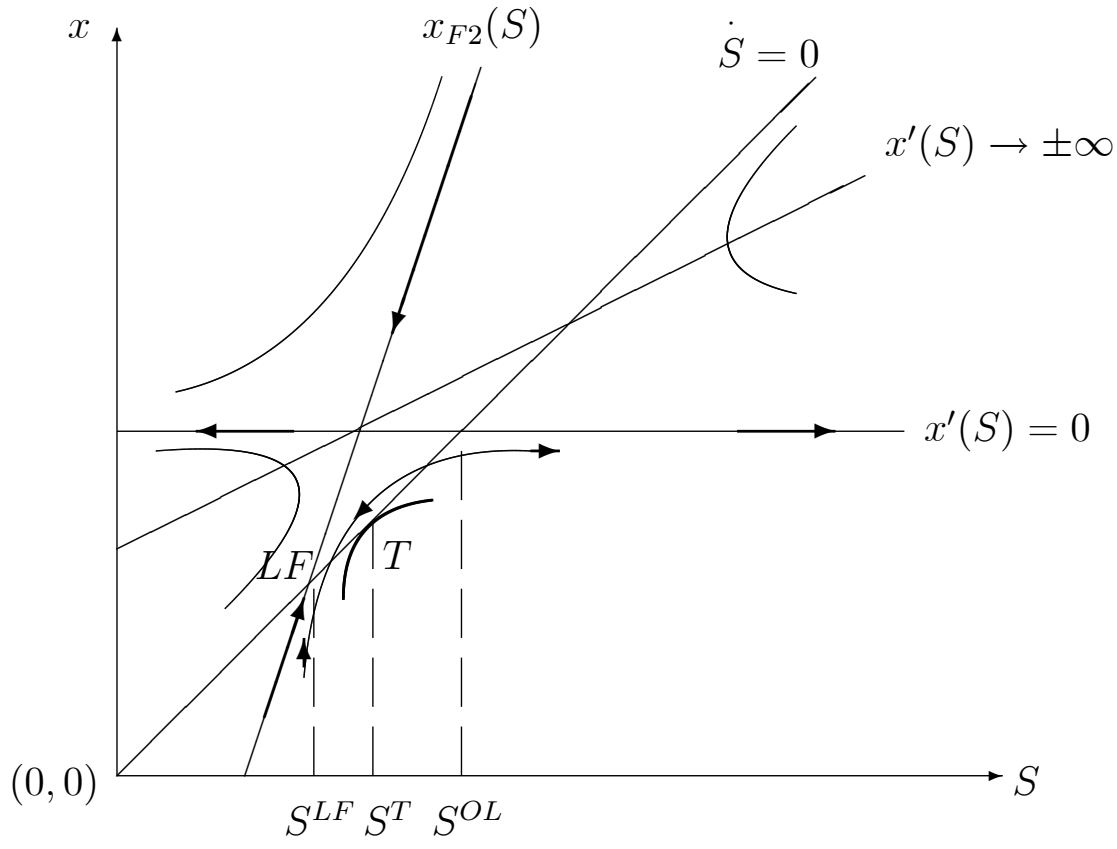

Changing the arbitrary value of $\boldsymbol{M}$ generates infinitely many nonlinear solutions. Figure 1 describes the evolution of the single state and the $n$ symmetric controls over time, allowing us to grasp the features of any nonlinear feedback solutions, including that generated by the tangency point with the locus $\dot{S}=0$ (point $T$ in the figure). Loci $x^{\prime}(S)=0$ (along which $\left.x_{0}(S)=x^{F 1}(S)=x^{O L}\right)$ and $x^{\prime}(S) \rightarrow \infty$ are also drawn. The arrows along the curve tangent to the locus $\dot{S}=0$ in point $T$ show that the tangency solution is indeed unstable. Nevertheless, there are infinitely many solutions identified by the intersections along the segment delimited by points $L F$ and $T$, which can be reached provided that the initial stock is sufficiently low to allow firms to locate themselves along a stable trajectory towards one of these points (see Itaya and Shimomura, 2001; Rubio and Casino, 2002). The 
length of $L F T$ is

$$
L F T=\sqrt{\left(S^{T}-S^{L F}\right)^{2}+\left(x^{T}-x^{L F}\right)^{2}}
$$

where $S^{L F}=S^{F 2}, S^{T}=n x^{T} / k$ and $x^{L F}=x^{F 2}$ at $S=S^{L F}$.

The foregoing analysis proves the following:

Proposition 1 For each $x \in\left[x^{L F}, x^{T}\right)$, there exists a unique $S \in\left[S^{L F}, S^{T}\right)$ solving $\dot{S}=0$. If $S_{0} \in\left(0, S^{T}\right)$, each of these pairs identifies a stable feedback solution of the game. The pair $\left(S^{L F}, x^{L F}\right)$ is the unique linear feedback solution, while the infinitely many others are nonlinear.

\section{Concluding remarks}

We have used the same approach as in Rowat (2007) to identify the continuum of nonlinear feedback equilibria arising in a game of duopolistic exploitation of a renewable resource initially investigated by Benchekroun (2003) and Fujiwara (2008). In doing so, we have also shown that the degenerate nonlinear tangency solution is indeed unstable. 


\section{References}

[1] Benchekroun, H. (2003). Unilateral production restrictions in a dynamic duopoly. Journal of Economic Theory 111, 214-39.

[2] Benchekroun, H. (2008). Comparative dynamics in a productive asset oligopoly. Journal of Economic Theory 138, 237-61.

[3] Cellini, R., Lambertini, L. and Leitmann, G. (2005). Degenerate feedback and time consistency in differential games, in E.P. Hofer and E. Reithmeier (eds), Modeling and Control of Autonomous Decision Support Based Systems. Proceedings of the 13th International Workshop on Dynamics and Control, Aachen, Shaker Verlag, 185-92.

[4] Colombo, L. and Labrecciosa, P. (2015). On the Markovian efficiency of Bertrand and Cournot equilibria. Journal of Economic Theory 155, $322-58$.

[5] Dockner, E.J., Jørgensen, S., Long, N.V. and Sorger, G. (2000). Differential Games in Economics and Management Science, Cambridge, Cambridge University Press.

[6] Dockner, E.J. and Sorger, G. (1996). Existence and properties of equilibria for a dynamic game on productive assets. Journal of Economic Theory 171, 201-27.

[7] Fershtman, C. (1987). Identification of classes of differential games for which the open-loop is a degenerate feedback Nash equilibrium", Journal of Optimization Theory and Applications, 55, 217-31.

[8] Fujiwara, K. (2008). Duopoly can be more anti-competitive than monopoly. Economics Letters 101, 217-19. 
[9] Itaya, J. and Shimomura, K. (2001). A dynamic conjectural variations model in the private provision of public goods: a differential game approach. Journal of Public Economics 81, 153-72.

[10] Lambertini, L. and Mantovani, A. (2014). Feedback equilibria in a dynamic renewable resource oligopoly: pre-emption, voracity and exhaustion", Journal of Economic Dynamics and Control, 47, 115-22.

[11] Mehlmann, A. (1988). Applied Differential Games, New York, Plenum Press.

[12] Rowat, C. (2007). Non-linear strategies in a linear quadratic differential game. Journal of Economic Dynamics and Control 31, 3179-202.

[13] Rubio, S.J., and Casino, B. (2002). A note on cooperative versus noncooperative strategies in international pollution control. Resource and Energy Economics 24, 251-61.

[14] Shimomura, K. (1991). The feedback equilibria of a differential game of capitalism. Journal of Economic Dynamics and Control 15, 317-38. 


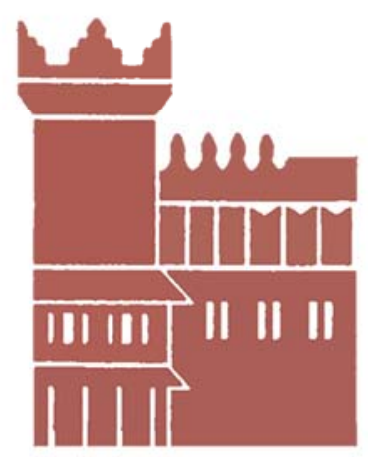

Alma Mater Studiorum - Università di Bologna DEPARTMENT OF ECONOMICS

Strada Maggiore 45

40125 Bologna - Italy

Tel. +39051 2092604

Fax +390512092664

http://www.dse.unibo.it 